\title{
Association of sagittal spinal alignment with thickness and echo intensity of lumbar back muscles in middle-aged and elderly women
}

\section{$\operatorname{AUTHOR}(\mathrm{S})$ :}

Masaki, Mitsuhiro; Ikezoe, Tome; Fukumoto, Yoshihiro; Minami, Seigo; Tsukagoshi, Rui; Sakuma, Kaoru; Ibuki, Satoko; Yamada, Yosuke; Kimura, Misaka; Ichihashi, Noriaki

\section{CITATION:}

Masaki, Mitsuhiro ... [et al]. Association of sagittal spinal alignment with thickness and echo intensity of lumbar back muscles in middle-aged and elderly women. Archives of Gerontology and Geriatrics 2015, 61(2): 197-201

\section{ISSUE DATE:}

2015-09

\section{URL:}

http://hdl.handle.net/2433/231299

\section{RIGHT:}

(c) 2015. This manuscript version is made available under the CC-BY-NC-ND 4.0 license

http://creativecommons.org/licenses/by-nc-nd/4.0/. The full-text file will be made open to the public on 01 September 2016 in accordance with publisher's 'Terms and Conditions for Self-Archiving'. This is not the published version. Please cite only the published version. この論文は出版社版でありません。引用の際には出版社版をご確認ご利用ください。 
Association of sagittal spinal alignment with thickness and echo intensity of lumbar back muscles in middle-aged and elderly women: A Cross-Sectional Study

Mitsuhiro Masaki ${ }^{1}$, Tome Ikezoe ${ }^{1}$, Yoshihiro Fukumoto², Seigo Minami ${ }^{3}$, Rui Tsukagoshi ${ }^{4}$, Kaoru Sakuma $^{1,5}$, Satoko Ibuki ${ }^{1}$, Yosuke Yamada ${ }^{6}$, Misaka Kimura $^{7}$, and Noriaki Ichihashi ${ }^{1}$

${ }^{1}$ Department of Physical Therapy, Human Health Sciences, Graduate School of Medicine, Kyoto University, Kyoto

53 Kawahara-cho, Shogoin, Sakyo-ku, Kyoto 606-8507, Japan.

${ }^{2}$ Department of Physical Therapy, Faculty of Rehabilitation, Kobe Gakuin University, Hyogo

518 Arise, Ikawadani-cho, Nishi-ku, Kobe 651-2180, Japan.

${ }^{3}$ Department of Occupational Therapy, Faculty of Allied Health Sciences, Yamato University, Osaka 2-5-1 Katayama-cho, Suita 564-0082, Japan.

${ }^{4}$ Department of Physical Therapy, Faculty of Rehabilitation, Hyogo University of Health Sciences, Hyogo

1-3-6 Minatojima, Chuo-ku, Kobe 650-8530, Japan.

${ }^{5}$ Japan Society for the Promotion of Science, Tokyo

5-3-1 Kojimachi-cho, Chiyoda-ku, Tokyo 102-0083, Japan. 
${ }^{6}$ Department of Nutritional Science, National Institute of Health and Nutrition, Tokyo

1-23-1 Toyama, Shinjyuku-ku, Tokyo 162-8636, Japan.

${ }^{7}$ Faculty of Bioenvironmental Science, Kyoto Gakuen University, Kyoto

1-1 Nanjyo-Otani, Sogabe-cho, Kameoka 621-8555, Japan.

Corressponding author

Mitsuhiro Masaki, R.P.T., M.Sc.

Department of Physical Therapy, Human Health Sciences,

Graduate School of Medicine, Kyoto University,

53 Kawahara-cho, Shogoin, Sakyo-ku, Kyoto 606-8507, Japan.

E-mail: masaki.mitsuhiro.27w@st.kyoto-u.ac.jp

Office phone: +81-75-751-3935

Office fax: +81-75-751-3909 


\section{Abstract}

Objective: Quantitative changes, such as a decrease in muscle mass, and qualitative changes, such as an increase in the amount of intramuscular non-contractile tissue, occur with aging. However, it is unclear whether quantitative or qualitative changes in back muscles are associated with spinal alignment in the standing position. We investigated the association of sagittal spinal alignment with muscle thickness as an index of the mass of lumbar back muscles and muscle echo intensity as an index of the amount of non-contractile tissue within these muscles.

Methods: Study participants comprised 36 middle-aged and elderly women. Thickness and echo intensity of erector spinae, psoas major, and lumbar multifidus muscles were measured using an ultrasound imaging device. Standing sagittal spinal alignment, determined from thoracic kyphosis and lumbar lordosis angles, and the sacral anterior inclination angle was measured using the Spinal Mouse.

Results: Stepwise regression analysis performed using muscle thickness, echo intensity, and age as independent variables showed that erector spinae muscle thickness was a significant determinant of the thoracic kyphosis angle. Psoas major muscle thickness and echo intensity of the lumbar multifidus muscle were significant determinants of the sacral anterior inclination angle.

Conclusion: Our results suggest that an increase in thoracic kyphosis is associated with a decrease in the mass of the erector spinae muscle, and that a decrease in pelvic anterior inclination is associated 
with a decrease in the mass of the psoas major muscle and an increase in the amount of non-contractile tissue within the lumbar multifidus muscle.

Key words: aged; middle-aged; paraspinal muscles; posture; ultrasonography 


\section{Introduction}

It is well established that age-related changes in spinal alignment in the standing position such as increased kyphosis (Kado et al., 2013; Takeda et al., 2009) and pelvic posterior inclination (Takeda et al., 2009) occur in middle-aged and elderly women. In fact, $20 \%-40 \%$ of elderly people develop hyperkyphosis, which may be caused by deformity of the vertebral body, degeneration of intervertebral disks, and muscle weakness (Kado, Prenovost, \& Crandall, 2007a). Hyperkyphosis leads to a decline in mobility, with effects such as decreased walking speed (Miyazaki et al., 2013; Katzman, Vittinghoff, \& Kado., 2011) and falls (Kado et al., 2007b). Therefore, improvement in spinal alignment and prevention of kyphosis progression are important for middle-aged and elderly people, especially in elderly women who have a higher risk of osteoporotic fracture (Swezey., 2000).

Although muscle weakness may contribute to hyperkyphosis alignment in the standing position, a previous study demonstrated no association of trunk flexor strength with lumbar lordosis and sacral anterior inclination angles (Kim et al., 2006). However, it has been revealed that a decrease in trunk extensor strength is associated with thoracic kyphosis and lumbar lordosis angles, and the sacral anterior inclination angle in the standing position (Sinaki et al., 1996). A previous study using computed tomography also indicates that the muscle density of lumbar back muscles including erector spinae and lumbar multifidus muscles is associated with thoracic kyphosis in the elderly individuals (Katzman et al., 2012). These studies suggest that kyphosis progression with aging may 
be associated with back muscles rather than abdominal muscles.

It has been verified that muscle thickness (MT) on ultrasound imaging, which is strongly correlated with muscle mass on magnetic resonance imaging (Miyatani et al., 2004), influences muscle strength (Fukumoto et al., 2012). Muscle strength is influenced by not only muscle quantity, such as muscle mass, but also muscle quality, such as the amount of intramuscular non-contractile tissue (i.e., adipose and fibrous tissue). It has been recently demonstrated that muscle echo intensity (EI) on ultrasound imaging, which is utilized as an objective assessment of muscle quality represents the amount of intramuscular non-contractile tissue (Pillen et al., 2009; Reimers et al., 1993). EI of upper and lower extremity muscles increases with aging (Arts., 2010; Ikezoe., 2012a) and these qualitative changes are associated with muscle strength in middle-aged and elderly women (Fukumoto et al., 2012).

With regard to a decrease in back muscle mass, the thickness of psoas major and erector spinae muscles in elderly women who are able to perform activities of daily living independently is lower than that in young women, whereas no difference is observed in the thickness of the lumbar multifidus muscle between the two groups (Ikezoe et al., 2011a,b; Ikezoe et al., 2012b). Thus, many studies investigated the effect of age on atrophy of back muscles. However, there have been few studies focusing on age-related qualitative changes in back muscles (McLoughlin et al., 1994). Furthermore, no study has individually evaluated quantitative or qualitative changes in back muscles, 
and examined whether their changes in each back muscle influence sagittal spinal alignment, i.e. thoracic kyphosis and lumbar lordosis angles, and the sacral anterior inclination angle in the standing position, in middle-aged and elderly women.

This study had two aims. First, we investigated the effect of age on MT as an index of the mass of lumbar back muscles and muscle EI as an index of the amount of non-contractile tissue within these muscles using ultrasound. Second, we examined the association of sagittal spinal alignment in the standing position with the thickness and EI of lumbar back muscles in middle-aged and elderly women.

\section{Participants and methods}

\subsection{Participants}

Study participants comprised 36 middle-aged and elderly women who were living independently in Kyoto, Japan. The participants were excluded if they had low back pain; a history of orthopedic, neurological, respiratory, or circulatory disorders; previous spinal surgery; or a history of low back pain lasting 3 months or more.

All participants provided written informed consent, and the protocol was approved by the Ethics Committee of the Kyoto University Graduate School and Faculty of Medicine. 


\subsection{Ultrasound measurement}

MT and EI were measured using a B-mode ultrasound imaging device (LOGIQ Book Xp; GE

Healthcare Japan, Tokyo, Japan) with an 8-MHz linear array probe. Longitudinal ultrasound images

of erector spinae and psoas major muscles and transverse ultrasound images of the lumbar multifidus

muscle were taken bilaterally in the prone position (Figure 1). Measurement sites were as follows:

erector spinae and psoas major muscles (Ikezoe et al., 2011a,b) were assessed $7 \mathrm{~cm}$ lateral from the L3 spinous process, and the lumbar multifidus muscle (Ikezoe et al., 2012) was assessed $2 \mathrm{~cm}$ lateral to the L4 spinous process. A 58-dB gain was used for all muscles, and dynamic focus depth was set to the depth of the muscles. Dynamic range $(69 \mathrm{~Hz})$ and time gain compensation in the neutral position were set for measurement.

From the obtained images, EI was determined using image processing software (ImageJ; National Institutes of Health, Bethesda, MD, USA). Regions of interest were set at the depth of 2.0-3.5 cm for the erector spinae muscle, $1.5-2.5 \mathrm{~cm}$ for the lumbar multifidus muscle, and $3.5-5.0 \mathrm{~cm}$ for the psoas major muscle, avoiding the surrounding fascia. The mean EI of the region was assessed by computer-assisted 8-bit gray-scale analysis and was expressed as a value between 0 (black) and 255 (white). Enhanced EI indicates an increase in the amount of intramuscular fibrous and adipose tissue. The mean values of the thickness and EI for the right and left muscles were used for statistical analyses. 
Furthermore, to examine the intrarater reliability of the ultrasound technique for measuring the thickness and EI of erector spinae, lumbar multifidus, and psoas major muscles, two images of each muscle were taken on two separate days in eight healthy volunteers (age, 23.5 \pm 1.5 years).

2.3. Measurement of spinal alignment in the standing position

Standing sagittal spinal alignment (thoracic kyphosis and lumbar lordosis angles, and the sacral anterior inclination angle) was measured using the Spinal Mouse (Index Ltd., Tokyo, Japan). The Spinal Mouse is an electronic computer-aided measuring device that measures intersegmental angles in a non-invasive manner. The Spinal Mouse was guided along the midline of the spine, starting at the spinous processus of C7 and finishing at S3. The thoracic kyphosis angle was calculated from the sum of 11 segmental angles from Th1/2 to Th11/12. The lumbar lordosis angle was calculated from the sum of six segmental angles from Th12/L1 to L5/S1. The sacral anterior inclination angle was calculated from the difference between the sacral angle and the vertical plane. Spinal alignment was measured three times, and the mean value was used for analyses. Previous studies (Kellis et al., 2008; Guermazi et al., 2006) demonstrated a high degree of intrarater reliability and validity for alignment measurement in the standing position using the Spinal Mouse.

\subsection{Statistical analyses}


Statistical analyses were performed using SPSS version 20.0 (IBM Japan; Tokyo, Japan). Spearman's correlation coefficient was used to investigate the relationship between MI, EI, spinal alignment, and age after each variable was evaluated using Shapiro-Wilk tests. Stepwise regression analysis, using MI, EI, and age as the independent variables, was employed to investigate associations with spinal alignment. The variance inflation factor (VIF) was computed to monitor for a multicollinearity effect. Furthermore, intraclass correlation coefficients [ICCs (1.1)] were calculated to examine intrarater reliabilities of MT and EI measurements. $P$ values of $<0.05$ were considered significant.

\section{Results}

Table 1 presents participant characteristics and MT, EI, and spinal alignment measurements in the standing position.

In the reliability analysis of MT measurement, ICC (1.1) values were 0.824 for the erector spinae muscle, 0.899 for the lumbar multifidus muscle, and 0.947 for the psoas major muscle. For EI measurement, ICC (1.1) values were 0.894 for the erector spinae muscle, 0.831 for the lumbar multifidus muscle, and 0.664 for the psoas major muscle.

Table 2 lists correlation coefficients between MT, EI, spinal alignment, and age. The lumbar lordosis angle showed a significant negative correlation with age, i.e., the lumbar lordosis angle 
decreased with age. MT, EI, and thoracic kyphosis and sacral anterior inclination angles were not significantly correlated with age.

Stepwise regression analysis revealed that only the thickness of the erector spinae muscle was a significant and independent determinant of the thoracic kyphosis angle, i.e., the thoracic kyphosis angle increased with a decrease in the thickness of the erector spinae muscle. The VIF value was 1.00. In stepwise regression analysis for the lumbar lordosis angle, no significant variable was found. The thickness of the psoas major muscle and EI of the lumbar multifidus muscle were significant and independent determinants of the sacral anterior inclination angle, i.e., the sacral anterior inclination angle decreased with a decrease in the thickness of the psoas major muscle and an increase in EI of the lumbar multifidus muscle. The VIF value was 1.01 (Table 3).

\section{Discussion}

Kyphosis progression with aging has been shown to be associated with balance in standing (Choi et al., 2011) and the risk of falling (Kado et al., 2007). However, the association of sagittal spinal alignment with the mass of lumbar back muscles and the amount of non-contractile tissue within these muscles has not been clarified. The present study examined the association of sagittal spinal alignment with MT as an index of the mass of lumbar back muscles and muscle EI as an index of the amount of non-contractile tissue within these muscles in middle-aged and elderly women. 
Our results showed that both the thickness and EI of lumbar back muscles were not significantly correlated with age. Ikezoe et al. (2011a,b; 2012) demonstrated that the thickness of erector spinae and psoas major muscles in elderly women is lower than that in young women. Our study targeted only middle-aged and elderly women, which may be the reason why no correlation with age was observed.

Stepwise regression analysis showed that the thoracic kyphosis angle increased with a decrease in the thickness of the erector spinae muscle. Antigravity muscles, such as the erector spinae muscle, which are located at the back of kyphosis thoracic vertebrae, play an important role in maintaining posture. Ikezoe et al. (2012) demonstrated that remarkable atrophy of antigravity muscles occurs in elderly women. Our results suggest that a decrease in the mass of the erector spinae muscle may influence thoracic kyphotic posture in middle-aged and elderly women. However, our result is inconsistent with the result indicating the association between muscle density of lumbar back muscles and thoracic kyphosis in the elderly individuals that previous study (Katzman et al., 2012) has demonstrated. The inconsistency of the results might be influenced by differences in study participants and in methods for measurement i.e. including erector spinae and lumbar multifidus muscles.

Although the lumbar lordosis angle showed a significant negative correlation with age, stepwise regression analysis showed that there was no association between the lumbar lordosis angle and both 
the thickness and EI of back muscles. Powell et al. (1986) demonstrated that the prevalence of lumbar disk degeneration reaches approximately $90 \%$ in elderly women aged $>71$ years. Furthermore, a longitudinal study (Takeda et al., 2009) revealed that a decrease in the lumbar lordosis angle is associated with lumbar disk anterior degeneration. Therefore, a decrease in the lumbar lordosis angle may be related to lumbar disk degeneration rather than the mass of lumbar back muscles or the amount of non-contractile tissue within these muscles.

Stepwise regression analysis also showed that the sacral anterior inclination angle decreased with a decrease in the thickness of the psoas major muscle and an increase in EI of the lumbar multifidus muscle. The psoas major muscle is an antigravity postural muscle. Ikezoe et al. (2011a) demonstrated that there is remarkable atrophy of the psoas major muscle in healthy elderly women compared with young women. Our results suggest that age-related atrophy might occur in the psoas major muscle, which may lead to decreased pelvic anterior inclination. The lumbar multifidus muscle also plays an important role in maintaining the upright posture of the pelvis (O'Sullivan et al., 2006). Previous studies (Ikezoe et al., 2012; McLoughlin et al., 1994) showed that the amount of adipose tissue within the lumbar multifidus muscle increases with aging, whereas the thickness of this muscle does not differ between elderly women who are able to perform activities of daily living independently and young women. Therefore, an age-related change in EI (i.e., an increase in the amount of non-contractile tissue within the lumbar multifidus muscle), which is greater than the 
change in MT, may lead to decreased pelvic anterior inclination.

The present study has several limitations. One limitation is that our MT and EI measurements targeted only lumbar back muscles. Another limitation is that this study was a cross-sectional study. Hyperkyphotic posture due to deformity of the vertebral body and degeneration of intervertebral disks with aging (Keorochana et al., 2011; Takeda et al., 2009) may lead to inactivity of lumbar back muscles, i.e., may cause changes in the thickness and EI of these muscles. However, it has been reported that strength training for back muscles is effective for improving hyperkyphotic posture (Katzman et al., 2007) or preventing kyphosis progress (Ball et al., 2009) in middle-aged and elderly women. Therefore, we assumed that the changes in the thickness and EI of lumbar back muscles may influence spinal alignment.

\section{Conclusions}

This study showed that sagittal thoracic and pelvic alignments in the standing position are associated with not only muscle quantity (i.e. muscle size) but also muscle quality (i.e. EI, the amounts of fibrous and adipose tissue within the muscle) of lumbar back muscles.

\section{Conflicts of interest statement}

No funding sources and potential conflicts of interest were disclosed for the present study. 


\section{Acknowledgments}

The authors would like to thank Saori Shibuta, Natsuki Yamakami, Kosuke Saida (Human Health

Sciences, Graduate School of Medicine, Kyoto University), and Junichi Aoyama (Kyoto Yawata

Hospital) for their practical and technical assistance. The authors also thank all study participants. 


\section{References}

Arts IM, Pillen S, Schelhaas HJ, Overeem S, Zwarts MJ. (2010). Normal values for quantitative muscle ultrasonography in adults. Muscle \& Nerve, 41, 32-41.

Ball JM, Cagle P, Johnson BE, Lucasey C, Lukert BP. (2009). Spinal extension exercises prevent natural progression of kyphosis. Osteoporosis International, 20, 481-489.

Choi CJ, Lim HW, Park MK, Cho JG, Im GJ, Chae SW. (2011). Does the kyphotic change decrease the risk of fall? Clincal Experimental Otorhinolaryngology, 4, 118-121.

Fukumoto Y, Ikezoe T, Yamada Y et al. (2012). Skeletal muscle quality assessed from echo intensity is associated with muscle strength of middle-aged and elderly persons. European Journal of Applied Physiology, 112, 1519-1525.

Guermazi M, Ghroubi S, Kassis M et al. (2006). Validity and reliability of Spinal Mouse to assess lumbar flexion. Annales de Réadaptation et de Médecine Physique, 49, 172-177

Ikezoe T, Mori N, Nakamura M, Ichihashi N. (2011a). Age-related muscle atrophy in the lower extremities and daily physical activity in elderly women. Archives of Gerontology and Geriatrics; 53, e153-157.

Ikezoe T, Mori N, Nakamura M, Ichihashi N. (2011b). Atrophy of the lower limbs in elderly women: is it related to walking ability? European Journal of Applied Physiology, 111, 989-995. 
Ikezoe T, Asakawa Y, Fukumoto Y, Tsukagoshi R, Ichihashi N. (2012a). Associations of muscle stiffness and thickness with muscle strength and muscle power in elderly women. Geriatrics \& Gerontology International, 12, 86-92.

Ikezoe T, Mori N, Nakamura M, Ichihashi N. (2012b). Effects of age and inactivity due to prolonged bed rest on atrophy of trunk muscles. European Journal of Applied Physiology, 112, 43-48.

Kado DM, Prenovost K, Crandall C. (2007a). Narrative review: hyperkyphosis in older persons. Annals of Internal Medicine; 147: 330-338.

Kado DM, Huang MH, Nguyen CB, Barrett-Connor E, Greendale GA. (2007b). Hyperkyphotic posture and risk of injurious falls in older persons: the Rancho Bernardo Study. The Journals of Gerontology. Series A, Biological Sciences and Medical Sciences, 62, 652-657.

Kado DM, Huang MH, Karlamangla AS et al. (2013). Factors associated with kyphosis progression in older women: 15 years' experience in the study of osteoporotic fractures. Journal of Bone and Mineral Research, 28, 179-187.

Katzman W, Cawthon P, Hicks GE et al. (2012). Association of spinal muscle composition and prevalence of hyperkyphosis in healthy community-dwelling older men and women. $J$ Gerontol A Biol Sci Med Sci, 67, 191-195. 
Katzman WB, Sellmeyer DE, Stewart AL, Wanek L, Hamel KA. (2007). Changes in flexed posture, musculoskeletal impairments, and physical performance after group exercise in community-dwelling older women. Archives of Physical Medicine and Rehabilitation, 88, 192-199.

Katzman WB, Vittinghoff E, Kado DM. (2011). Age-related hyperkyphosis, independent of spinal osteoporosis, is associated with impaired mobility in older community-dwelling women. Osteoporosis International, 22, 85-90.

Kellis E, Adamou G, Tzilios G, Emmanouilidou M. (2008). Reliability of spinal range of motion in healthy boys using a skin-surface device. Journal of Manipulative and Physiological Therapeutics; 31, 570-576.

Keorochana G, Taghavi CE, Lee KB et al. (2011). Effect of sagittal alignment on kinematic changes and degree of disc degeneration in the lumbar spine: an analysis using positional MRI. Spine (Phila Pa 1976), 36, 893-898.

Kim HJ, Chung S, Kim S, Shin H, Lee J, Song MY. (2006). Influences of trunk muscles on lumbar lordosis and sacral angle. European Spine Journal, 15, 409-414.

McLoughlin RF, D'Arcy EM, Brittain MM, Fitzgerald O, Masterson JB. (1994). The significance of fat and muscle areas in the lumbar paraspinal space: a CT study. Journal of Computer Assisted Tomography, 18, 275-278. 
Miyatani M, Kanehisa H, Ito M, Kawakami Y, Fukunaga T. (2004). The accuracy of volume estimates using ultrasound muscle thickness measurements in different muscle groups. European Journal of Applied Physiology, 91, 264-272.

Miyazaki J, Murata S, Horie J, Uematsu A, Hortobágyi T, Suzuki S. (2013). Lumbar lordosis angle (LLA) and leg strength predict walking ability in elderly males. Archives of Gerontology and Geriatrics, 56, 141-147.

O'Sullivan PB, Dankaerts W, Burnett AF et al. (2006). Effect of different upright sitting postures on spinal-pelvic curvature and trunk muscle activation in a pain-free population. Spine (Phila Pa 1976), 31, E707-712.

Pillen S, Tak RO, Zwarts MJ et al. (2009). Skeletal muscle ultrasound: correlation between fibrous tissue and echo intensity. Ultrasound in Medicine \& Biology, 35, 443-446.

Powell MC, Wilson M, Szypryt P, Symonds EM, Worthington BS. (1986). Prevalence of lumbar disc degeneration observed by magnetic resonance in symptomless women. The Lancet, 2, 1366-1367.

Reimers K, Reimers CD, Wagner S, Paetzke I, Pongratz DE. (1993). Skeletal muscle sonography: a correlative study of echogenicity and morphology. Journal of Ultrasound in Medicine, 12, 73-77.

Sinaki M, Itoi E, Rogers JW, Bergstralh EJ, Wahner HW. (1996). Correlation of back 
extensor strength with thoracic kyphosis and lumbar lordosis in estrogen-deficient women.

American Journal of Physical Medicine \& Rehabilitation, 75, 370-374.

Swezey RL. Osteoporosis: diagnosis, pharmacological, and rehabilitation therapies. (2000).

Critical Reviews in Physical and Rehabilitation Medicine, 12, 229-269.

Takeda N, Kobayashi T, Atsuta Y, Matsuno T, Shirado O, Minami A . (2009). Changes in the sagittal spinal alignment of the elderly without vertebral fractures: a minimum 10-year longitudinal study. Journal of Orthopaedic Science, 14, 748-753. 
Table 1. Characteristics and muscle thickness, muscle echo intensity, and spinal alignment measurements in healthy middle-aged and elderly female participants.

\begin{tabular}{|c|c|c|}
\hline & Mean \pm SD & Range \\
\hline \multicolumn{3}{|l|}{ Characteristics } \\
\hline Age (years) & $72.4 \pm 8.0$ & $54.0-91.0$ \\
\hline Height (cm) & $150.2 \pm 4.5$ & $140.1-161.0$ \\
\hline Weight (kg) & $48.8 \pm 7.7$ & $37.4-65.5$ \\
\hline \multicolumn{3}{|l|}{ MT (cm) } \\
\hline Erector spinae & $2.23 \pm 0.48$ & $1.22-3.20$ \\
\hline Lumbar multifidus & $2.34 \pm 0.35$ & $1.29-2.95$ \\
\hline Psoas major & $1.40 \pm 0.40$ & $0.80-2.43$ \\
\hline \multicolumn{3}{|l|}{ EI } \\
\hline Erector spinae & $71.1 \pm 12.1$ & 49.8-101.5 \\
\hline Lumbar multifidus & $77.3 \pm 7.4$ & $61.5-90.2$ \\
\hline Psoas major & $41.0 \pm 9.7$ & $21.1-60.1$ \\
\hline \multicolumn{3}{|l|}{ Spinal alignment $\left({ }^{\circ}\right)$} \\
\hline Thoracic kyphosis & $36.1 \pm 13.7$ & $13.0-86.0$ \\
\hline Lumbar lordosis & $12.7 \pm 7.1$ & -10.0 to 24.0 \\
\hline
\end{tabular}


MT: muscle thickness, EI: muscle echo intensity, SD: standard deviation 
Table 2. Relationships between muscle thickness, muscle echo intensity, spinal alignment, and age.

\section{Age}

Erecter spinae MT

0.07

Lumbar multifidus MT

$-0.02$

Psoas major MT

$-0.14$

Erecter spinae EI

$-0.09$

Lumbar multifidus EI

$-0.21$

Psoas major EI

0.14

Thoracic kyphosis

$-0.16$

Lumbar lordosis

$-0.34^{*}$

Sacral anterior inclination

$-0.04$

${ }^{*} P<0.05$

MT: muscle thickness, EI: muscle echo intensity 
Table 3. Results of stepwise regression analyses.

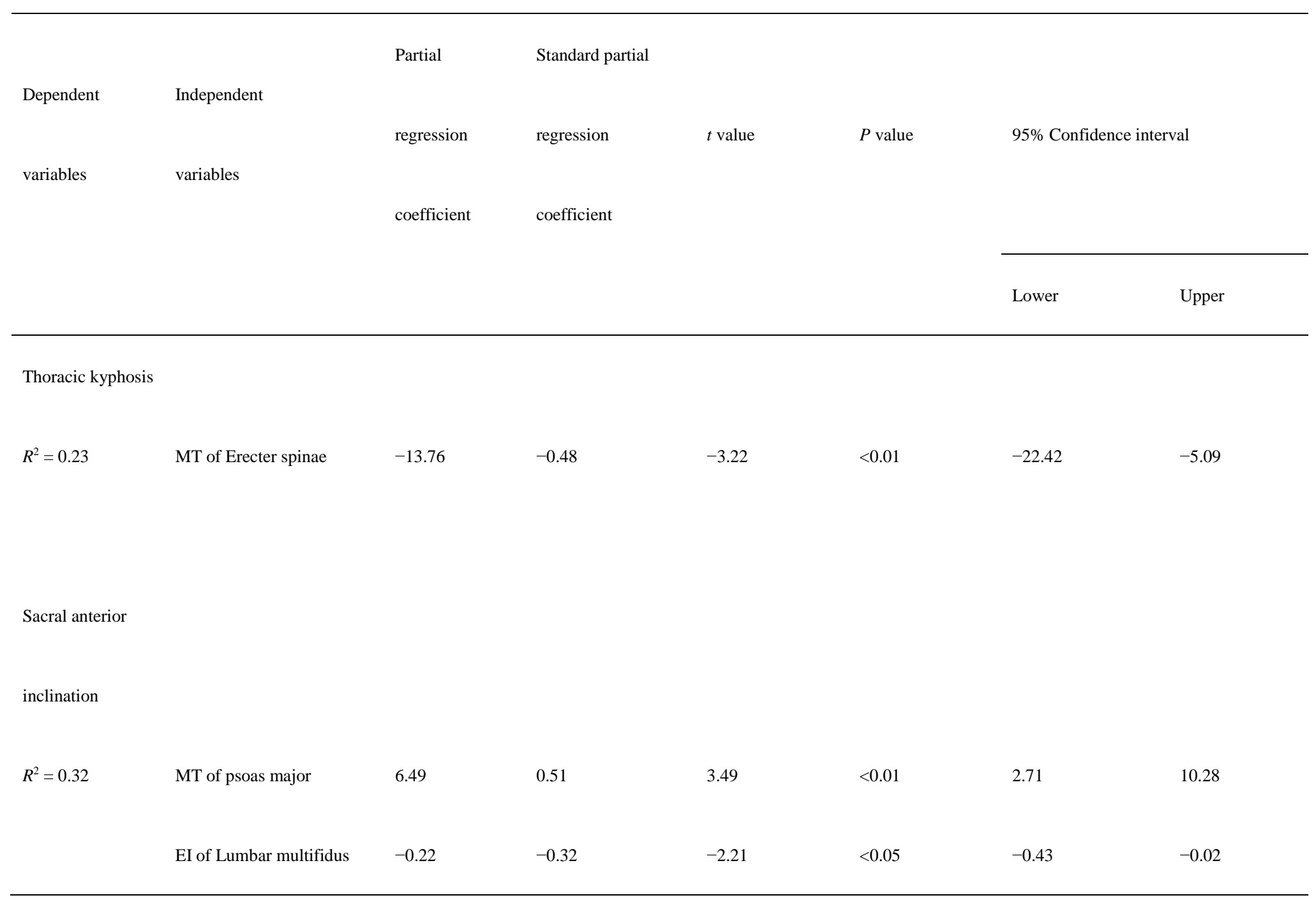

MT: muscle thickness, EI: muscle echo intensity, $R^{2}$ : Coefficient of determination 


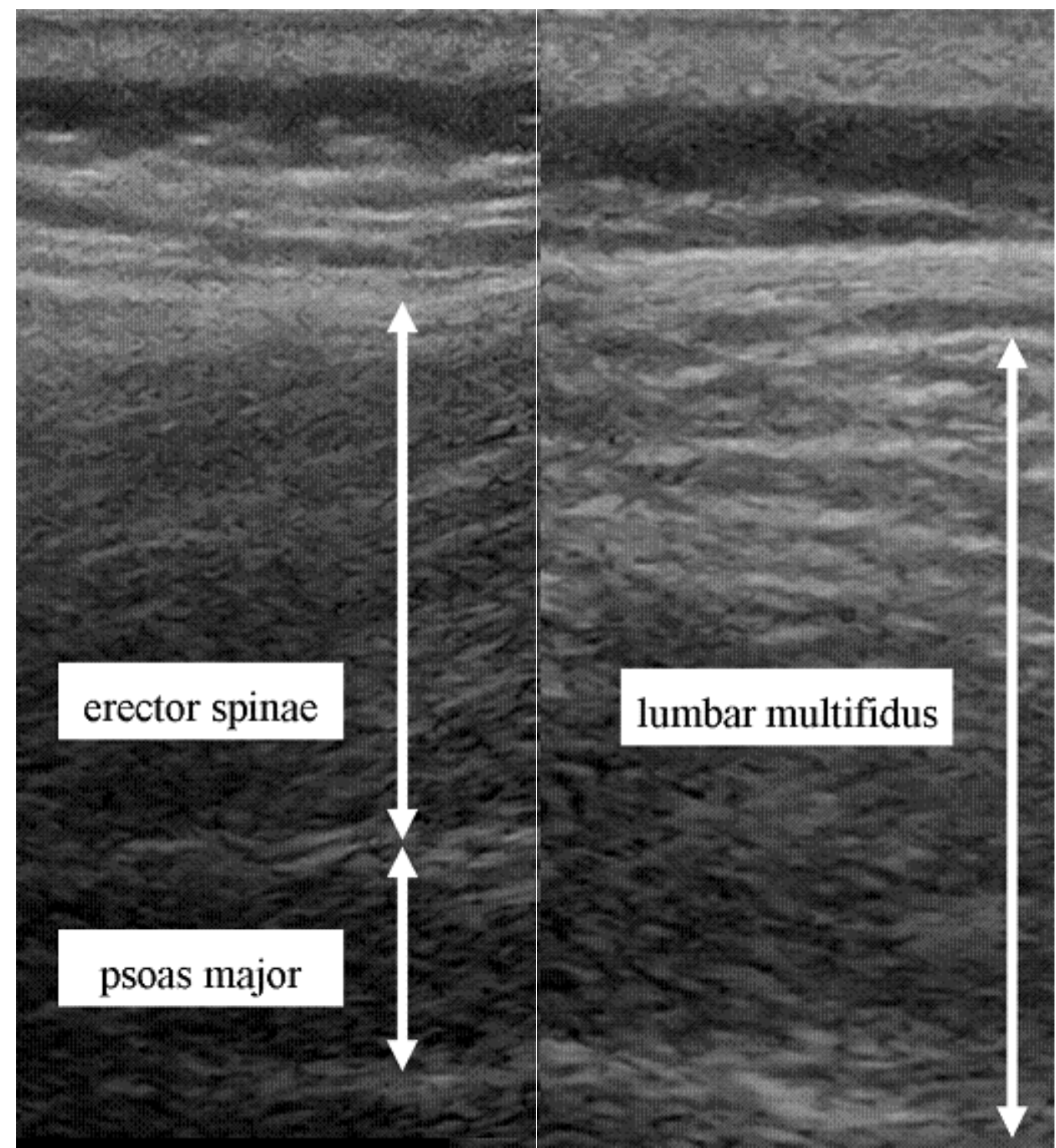

Figure 1. Representative ultrasound images of lumbar back muscles. 